When L-tyrosine alone was added to the diet $(0.6 \mathrm{~g}$. the first day and $2.25 \mathrm{~g}$. the next two days) the fasting serum tyrosine increased to $5 \mathrm{mg} . / 100 \mathrm{ml}$., the ferric chloride and D.P.H. tests in the urine got strongly positive, the serum phosphorus dropped to 1.8 and $2 \mathrm{mg} . / 100 \mathrm{ml}$., and the T.R.P. decreased to $10 \%$ (see Chart). The excretion of the amino-acids and the p-hydroxyphenolic acids increased again to levels as before the diet (see Table).

The mother, who stayed with the child during the dietary period, thought her child was more active and alert during the last part of the low-tyrosine regimen than she had ever been, but this was not apparent to other observers.

\section{Discussion}

The clinical disorder described in this paper and previously reported by Sakai and Kitagawa (1957a, 1957b), Sakai et al. (1959), Zetterström (1963), and Fritzell et al. (1964) has characteristic clinical, biochemical, and morphological findings, and it is our opinion that it represents a disease entity. Although the ultimate proof is still lacking, the previously reported findings and the data here presented support the hypothesis that the disorder is caused by a deficiency of p-hydroxyphenyl-pyruvate oxidase. An inhibition of this enzyme rather than a genetically determined lack cannot be excluded, but we accept Zetterström's view that the disease may be termed "tyrosinosis" rather than atypical tyrosinosis as called by Sakai et al.

The results of the low-tyrosine diet in the above case indicate that the high levels of tyrosine and/or its metabolites are of fundamental importance for the development of the disease. The relation between the levels of tyrosine and its metabolites and the renal handling of phosphorus and amino-acids suggests a causal effect. Although it may be argued that the increased phosphorus reabsorption on a low-tyrosine diet could be caused by extrarenal factors, the simultaneous increase in amino-acid reabsorption indicates that it has had an effect on the renal tubules. The definite decrease in renal epithelial cells and renal casts also supports this assumption. The mechanism by which tyrosine or its metabolites may increase phosphorus and aminoacid excretion remains unknown, but it is likely that the tubular transport of these compounds is inhibited. It is also interesting that glucose transport is less affected, since the patient only occasionally had glycosuria.

The effect of the low-tyrosine diet on the thrombocytes is less convincing, and in this short trial the liver function has not been affected, judged by the P.P. test. This was, however, not expected, since the patient already had a far-advanced liver cirrhosis. Although the present study was promising, the ultimate effect of the low-tyrosine diet in this disease cannot yet be assessed. We must await further trials, both long-term and earlier in life, before liver damage has occurred. These preliminary results are published in order to stimulate others to try the diet in such cases.

The above study indicates that the intake of phenylalanine and tyrosine must be low in order to normalize the metabolism. An intake of phenylalanine and tyrosine of about $25 \mathrm{mg}$. of each per kg. body weight per day lowered serum tyrosine to $1.5 \mathrm{mg} . / 100 \mathrm{ml}$., and it was at this level that the improvement in renal function first became convincing.

\section{Summary}

A case of tyrosinosis in a 2-year-old girl is described. The serum-tyrosine level was raised and the urinary excretion of p-hydroxyphenyl-pyruvic acid, p-hydroxyphenyl-lactic acid, and $p$-hydroxyphenyl-acetic acid was much increased. She had marked hyperphosphaturia and hyperaminoaciduria. A four-weeks trial with a low-tyrosine and low-phenylalanine diet decreased the serum-tyrosine level while the serum-phosphorus values increased. The excretion of the tyrosine metabolites, amino-acids, and phosphorus in the urine decreased and the excretion of renal epithelial cells and renal casts diminished. On adding L-tyrosine to the low-tyrosine and low-phenylalanine diet the serum tyrosine again increased while the serum phosphorus decreased, and there was an increase in the excretion of the tyrosine metabolites, amino-acids, and phosphorus in the urine. These findings support the hypothesis that this disease is caused by a defect of $p$-hydroxyphenyl-pyruvate oxidase, and indicate that tyrosine or its metabolites have a " toxic" effect on renal tubular cells and possibly on liver cells.

\section{REFERENCES}

Armstrong, M. D., Shaw, K. N. F., and Wall, P. E. (1956). F. biol. Chem., 218, 293.

Berry, H. K. (1962). Clin. Chem., 8, 172.

Fritzell, S., Jagenburg, O. R., and Schnürer, L. B. (1964). Acta paediat. (Stockh.), 53, 18.

Goldman, L., Gordan, G. S., and Chambers, E. L. (1957). Ann. Surg., $146,407$.

Medes, G. (1932). Biochem. 7., 26, 917.

Owren, P. A., and Aas, K. (1951). Scand. f. clin. Lab. Invest., 3, 201.

Sakai, K., and Kitagawa, T. (1957a). fikeikai med. f., 4, 1.

- (1957b). Ibid., 4, 11.

Zetterström, R. (1963). Ann. N.Y. Acad. Sci., 11i, 220.

\title{
Evaluation of Immunofluorescent and Direct Agglutination Methods for Detection of Specific Toxoplasma Antibodies
}

\author{
J. D. FULTON,*† M.A., M.B., D.T.M. ; A. VOLLER, ${ }^{*} \ddagger$ M.SC., PH.D.
}

A number of serological tests have been described for the detection of antibodies to Toxoplasma gondii, the most recent being the fluorescent antibody test (F.A.T.). (Goldman, 1957 ; Kelen et al., 1962 ; Mandras et al., 1962 ; Camargo, 1964 ; Kramarzh, 1963). We had at our disposal a number of sera whose titres had been determined by the dye test (Sabin and Feldman, 1948), the complement-fixation test (C.F.T.) (Fulton and Dumbell, 1949), and the direct agglutination test (D.A.T.) (Fulton and Turk, 1959). It was possible, therefore, to compare the results with those determined by the newer method.

\section{Materials and Methods}

The antigen, prepared by the method of Fulton and Turk (1959), was a suspension of $T$. gondii free from other cells, except an occasional red cell. Smears were made on microscope slides, which were then dried for several hours in a desiccator before being transferred for storage at $-70^{\circ} \mathrm{C}$. These smears

* London School of Hygiene and Tropical Medicine.

† Supported by the Medical Research Council.

F Supported by the World Health Organization. 
were suitably reactive even after several months' storage. Sufficient serum for the test could even be obtained from finger-prick blood, using microhaematocrit tubes for the sample.

The method of fluorescent staining was the same as that used for malaria parasites by Voller (1964). The antigen smears are treated with $\mathrm{N} / 3$ hydrochloric acid, then the test serum, and finally the fluorescein-labelled anti-human-globulin serum. The first examination was made with the test serum diluted $1: 20$, and, if positive, further twofold dilutions were made in order to determine the end-point. The end-point, although arrived at by a subjective estimation, was usually quite clear, being the highest dilution at which distinct staining of the organisms occurred. All examinations of slides were carried out on a Reichert fluorescence microscope using ultra-violet-blue excitation in combination with a bright-field condenser.

In order to verify the specificity of the test, absorption studies were carried out with the preparations of $T$. gondii used as antigen, Trypanosoma rhodesiense, Leishman-Donovan bodies of Leishmania donovani, and kieselguhr or sugars.

The usual weight of parasites used was 7-10 mg. of dried material per millilitre of serum diluted $1: 10$, and absorption was carried out overnight. The preliminary tests were carried out on the sera of patients believed to be free from other infections. Sera from patients with other parasitic diseases, including malaria, filariasis, schistosomiasis, sleeping sickness, kala-azar, leptospirosis, and syphilis, were also tested. Particularly interesting was a single serum from a patient infected with Sarcocystis. ${ }^{1}$ Human sarcosporidiosis is a rarely diagnosed disease, and in the past the organism has often been confused with Toxoplasma.

\section{Results}

It is clear from the results in Table I, even with this limited number of sera tested, that there is a marked correlation in the titres obtained by the different methods. They seldom differ from one another by more than one tube in a series of twofold dilutions, and the agreement regarding positive and negative sera is excellent.

Table I.-Titration of Toxoplasma Antibodies in the Sera of Patients Apparently Free from Other Infections

\begin{tabular}{|c|c|c|c|c|}
\hline Serum No. & F.A.T. & Dye Test & D.A.T. & C.F.T. \\
\hline $\begin{array}{r}1 \\
2 \\
3 \\
4 \\
5 \\
6 \\
7 \\
8 \\
9 \\
10 \\
11 \\
12 \\
13 \\
14 \\
15 \\
16 \\
17 \\
18 \\
19 \\
20\end{array}$ & $\begin{array}{r}\text { Neg. } \\
128 \\
128 \\
2,048 \\
512 \\
8 \\
\text { Neg. } \\
\text { ” } \\
\text { ”, } \\
16 \\
128 \\
64 \\
128 \\
64 \\
4,096 \\
4,096 \\
4,096 \\
2,048 \\
256\end{array}$ & $\begin{array}{c}\text { Neg. } \\
128 \\
256 \\
1,024 \\
1,024 \\
<8 \\
\text { Neg. } \\
\text { ” } \\
\text { "” } \\
128 \\
128 \\
256 \\
256 \\
4,096 \\
4,096 \\
16,384 \\
1,024 \\
2,048\end{array}$ & $\begin{array}{r}16 \\
256 \\
1,024 \\
2,048 \\
1,024 \\
16 \\
8 \\
<8 \\
8 \\
8 \\
8 \\
128 \\
256 \\
1,024 \\
256 \\
2,048 \\
8,192 \\
8,192 \\
1,024 \\
2,048\end{array}$ & $\begin{array}{r}\text { N.T. } \\
\text { ", } \\
\text { ", } \\
\text { "” } \\
8 \\
8 \\
8 \\
16 \\
\text { Neg. } \\
64 \\
64 \\
32 \\
16 \\
512 \\
>1,024 \\
2,048 \\
1,024 \\
1,024\end{array}$ \\
\hline
\end{tabular}

The figures in this and other tables represent the reciprocal of serum dilutions a the end-point. N.T. = Not tested. F.A.T. = Fluorescent antibody test.

We then sought to establish the specificity of the fluorescent staining method, ( $a$ ) by using sera from patients with different infections, and $(b)$ by absorption studies. The results are shown in Tables II-VI.

Absorption of sleeping sickness sera (Table II) was also carried out with a suspension of trypanosomes containing the same weight of parasites as in the experiment using $T$. gondii ${ }^{1}$ We are indebted to Dr. A. Mandour for providing this serum and the
sarcocystis antigen. for absorption. There was no significant reduction of titre when trypanosomes were the absorbing agent.

All the sera in Table III had high F.A.T. titres for malaria antibody. The confirmation that the Toxoplasma antibody titres were due to Toxoplasma was given by absorption of the sera with $T$. gondii suspensions, which produced a marked reduction of titres. This reduction did not occur when kieselguhr was used as the absorbing reagent.

TABLE II.-Titration of Toxoplasma Antibodies in the Sera of Patients with Sleeping Sickness. Results Show Titres Both Before and After Absorption with $T$. gondii

\begin{tabular}{c|c|c|c|c}
\multirow{2}{*}{$\begin{array}{c}\text { Serum } \\
\text { No. }\end{array}$} & \multicolumn{2}{|c|}{ Titre before Absorption } & \multicolumn{2}{c}{ Titre After Absorption } \\
\cline { 2 - 5 } \cline { 3 - 4 } & F.A.T. & D.A.T. & F.A.T. & D.A.T. \\
\hline 1 & 40 & 160 & $<20$ & 80 \\
2 & 20 & 160 & Neg. & 10 \\
3 & 160 & 1,280 & 20 & 160 \\
4 & 160 & 320 & Neg. & 20 \\
5 & 80 & 1,280 & 20 & 80 \\
6 & 80 & 640 & Neg. & 160 \\
7 & 320 & 640 & 20 & 80 \\
8 & 640 & 1,280 & 40 & 160 \\
9 & 40 & 320 & 20 & 40 \\
10 & 40 & 320 & 40 & 40 \\
11 & 80 & 320 & 20 & 80 \\
12 & 160 & 640 & 20 & 20 \\
13 & 80 & 640 & Neg. & 20 \\
\hline
\end{tabular}

TABLE III.-Titration of Toxoplasma Antibodies in the Sera of Patients with Malaria

\begin{tabular}{c|c|c}
\hline Serum No. & Titre F.A.T. & Titre D.A.T. \\
\hline 1 & 80 & 160 \\
2 & 40 & 160 \\
3 & 160 & 640 \\
4 & 160 & 160 \\
5 & $<20$ & 40 \\
6 & 80 & 640 \\
\hline
\end{tabular}

TABLE IV.-Titration of Toxoplasma Antibodies in the Sera of Patients with Filariasis

\begin{tabular}{c|r|r|r|r}
\hline \multirow{2}{*}{$\begin{array}{c}\text { Serum } \\
\text { No. }\end{array}$} & \multicolumn{2}{|c|}{ Titre Before Absorption } & \multicolumn{2}{c}{ Titre After Absorption } \\
\cline { 2 - 3 } \cline { 4 - 5 } & F.A.T. & D.A.T. & $\begin{array}{c}\text { T. gondii } \\
\text { D.A.T. }\end{array}$ & $\begin{array}{c}\text { Kieselguhr } \\
\text { D.A.T. }\end{array}$ \\
\hline 1 & 80 & 160 & 10 & 160 \\
2 & 80 & 160 & 10 & 80 \\
3 & 20 & 80 & 10 & 80 \\
4 & 320 & 1,280 & 160 & 1,280 \\
5 & 320 & 640 & 10 & 320 \\
6 & 320 & 160 & 10 & 160 \\
7 & & 1,280 & 160 & 1,280 \\
\hline
\end{tabular}

The presence of specific antibodies is indicated by the results of the absorption tests in which $T$. gondii absorption reduced titres, whereas absorption with kieselguhr did not (Table IV).

When experiments with F.A.T. were started, only three sera were available (Table V). However, a total of 23 kala-azar sera were tested earlier by D.A.T. These gave titres in the range of normal uninfected patients. Absorption with $T$. gondii

Table V.-Titration of Toxoplasma Antibodies in the Sera of Patients with Kala-azar

\begin{tabular}{c|c|c}
\hline Serum No. & Titre F.A.T. & Titre D.A.T. \\
\hline 1 & 2,560 & 10,240 \\
2 & 20 & 160 \\
3 & $<20$ & 40
\end{tabular}

Table VI.-Titration of Toxoplasma Antibodies in the Sera of Patients with Leptospirosis

\begin{tabular}{c|c|c}
\hline Serum No. & Titre F.A.T. & Titre D.A.T. \\
\hline 1 & 20 & 160 \\
2 & 40 & 160 \\
3 & 20 & 80 \\
4 & 20 & 160 \\
5 & 20 & 40 \\
6 & 160 & 40 \\
7 & 80 & 640 \\
8 & 20 & 640 \\
9 & 20 & 160 \\
10 & & 160 \\
\hline
\end{tabular}


suspensions markedly reduced the titres in both tests, whereas absorption with Leishman-Donovan bodies from infected hamster spleens failed to reduce the titres.

Absorption experiments were carried out on sera 7-10 (Table VI) with $T$. gondii or with xylan. Only the former caused a reduction in titres, thus indicating the specific nature of the tests.

One serum from a patient with schistosomiasis and another from a syphilitic patient had very low titres, indicating that cross-reacting antibodies to Toxoplasma were not present.

Although diagnosis of sarcosporidiosis is common in sheep, it is extremely rare in man during life (Mandour, 1964). There

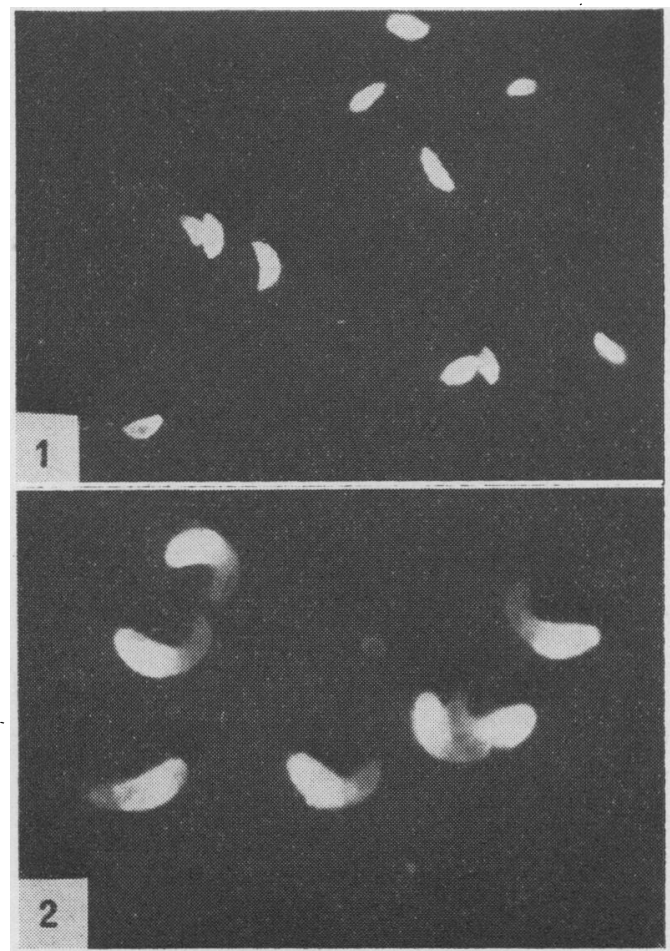

Fluorescence photomicrographs of parasites stained by the indirect fluorescent antibody method. In each case the homologous human serum was used, followed by fluorescein-labelled anti-human-globulin serum. 1, Toxoplasma gondii. 2, Sarcocystis tenella.

has been controversy in the past on whether there are crossreactions between this disease and toxoplasmosis. We were fortunate in obtaining serum from a patient in whom a diagnosis of Sarcocystis infection had been confirmed by biopsy. The serum had a high F.A.T. titre with Sarcocystis antigen, but was negative with Toxoplasma antigen. Similarly, high Toxoplasma F.A.T. titre sera did not react with Sarcocystis, nor were there cross-reactions in the D.A.T. series. This confirms the general consensus of opinion, which is that these organisms are immunologically distinct. The appearances of the two organisms, stained with homologous serum by the indirect F.A.T., are given in the illustration.

\section{Discussion}

It is clear that there is a considerable correlation between the different tests used in the present studies to indicate antibodies to Toxoplasma. The results obtained from the F.A.T. and D.A.T., and by absorption studies, on the sera of patients suffering from a range of parasitic infections, bear witness to the specificity of these serological tests. Goldman et al. (1957) noted that, using F.A.T., there was no cross-reaction with Besnoitia jellisoni. This fits in well with our lack of crossreactions with Sarcocystis.

It appears that the D.A.T. is slightly more sensitive than the F.A.T. In our test groups of sera the range of titres, although somewhat high, is well below that of patients known to be infected with Toxoplasma.

Although the dye test of Sabin and Feldman (1948) is the most widely used for Toxoplasma antibodies, it is rather difficult to set up and carry out. The D.A.T. has the advantage of being macroscopic, easy to perform, and only dead parasites are used. Data of a large-scale comparison between the above two tests will be available soon. The use of the pure agglutination test antigen for the complement-fixation test has made possible the exact comparison of results from different laboratories, which in the past has been difficult because of the heterogeneous nature of the antigens used. Goldman (1957) used a fluorescent inhibition test, but this required the preparation of a fluoresceinlabelled anti-Toxoplasma serum. The indirect F.A.T. which we have used has the advantage that all the reagents are commercially available, and that slide antigen can be easily prepared and stored for long periods. It is not quite so sensitive as the direct agglutination technique and fewer samples can be conveniently tested at any one time. In addition, special fluorescent equipment is necessary, although many laboratories are now equipped with this. The F.A.T. methods could prove of value in survey work to assess serum samples from finger-prick blood for detection of Toxoplasma antibody at a certain dilution.

\section{Summary}

Toxoplasma antibodies in human sera have been quantitatively assessed by indirect fluorescent antibody staining. The results obtained correlate well with those obtained by the dye test, by the direct-agglutination test, and by complement fixation. The fluorescent method is slower, but its specificity has been established by absorption studies and by lack of cross-reaction with sera from cases of trypanosomiasis, malaria, kala-azar, sarcosporidiosis, leptospirosis, syphilis, schistosomiasis, and filariasis.

\section{REFERENCES}

Camargo, M. E. (1964). Rev. Inst. Med. trop. S. Paulo, 6, 117

Fulton, F., and Dumbell, K. R. (1949). f. gen. Microbiol., 3, 97. Fulton, J. D., and Turk, J. L. (1959). Lancet, 2, 1068. Goldman, M. (1957). f. exp. Med., 105, 557.

Carver, R. K., and Sulzer, A. J. (1957). ₹. Parasit., 43, 490. Kelen, A. E., Ayllon-Leindl, L., and Labzoffsky, N. A. (1962). Canad.
f. Microbiol., 8, 545.

Kramarzh, Y. (1963). Med. Parazit. (Mosk.), 32, 454.

Mandour, A. M. (1964). Trans. roy. Soc. trop. Med. Hyg., 58, 287. Mandras, A., Vanini, G. C., and Ciarlini, E. (1962). Igiene mod., 56, 636.

Sabin, A. B., and Feldman, H. A. (1948). Science, 108, 660.

Voller, A. (1964). Bull. Wld Hith Org., 30, 343. 